\title{
Context-aware Load Balancing for Heterogeneous Smart-Grid Communication Networks
}

\author{
Yintao $\mathrm{Li}^{1, \mathrm{a}}$, Weiguo Yuan ${ }^{1, \mathrm{~b}}$, Hongfei $\mathrm{Xu}^{1, \mathrm{c}}$, Wei Song ${ }^{1, \mathrm{~d}}$ \\ , Rui Yang ${ }^{1, \mathrm{e}}$, Zhibin Zang ${ }^{2, \mathrm{f}}$, Dapeng $\mathrm{Lin}^{2, \mathrm{~g}}$ and Yeshen $\mathrm{He}^{2, \mathrm{~h}}$ \\ ${ }^{1}$ State Grid Jibei Information \& Telecommunication Company \\ ${ }^{2}$ NARI Group, China Gridcom CO,.LTD ,Shenzhen ,Guangdong,China \\ a734860195@qq.com, bwgy83866@sina.com, ${ }^{\mathrm{c}} 21147341 @ q q . c o m$, \\ d15910336689@163.com, 'lai_en@foxmail.com, ${ }^{\dagger}$ zangzhibin@sgepri.sgcc.com.cn, \\ glindapeng@sgepri.sgcc.com.cn, hheyeshen@sgepri.sgcc.com.cn
}

\begin{abstract}
Keywords:load balancing; context-aware; heterogeneous wireless networks; radio resource management; smart grid.
\end{abstract}

\begin{abstract}
The network of smart grid communications (SGC) is expected to be heterogeneous for providing diverse access interfaces. Load balancing is one of the key technologies in such heterogeneous networks, which can increase the system throughput as well as quality of service (QoS). In this paper, a context-aware load-balancing scheme is proposed for SGC networks by virtue of an adaptive trigger threshold. We investigate the characteristics of different kinds of services in the wireless local access network (WLAN) and Long Term Evolution (LTE). Simulation results show that our proposed load balance algorithm achieves better fairness and thus improves the overall throughput.
\end{abstract}

\section{Introduction}

The public-utility industry envisions the traditional power grid to incorporate the advanced communication systems for improving the control, efficiency, reliability and safety of power distribution and resource management. The resulting next-generation electric grid, known as smart grid, is expected to support a myriad of applications that have heterogeneous traffic requirements. Continuously increasing the demand of bandwidth, the intensive applications and the users' variable appetite for services of smart grid lead to a tremendous pressure to the smart grid communication (SGC) systems. To address the wide variety of applications, scenarios, regions, environments and topologies in smart grid, multiple radio access technologies (RATs) such as wireless local access network (WLAN), Long Term Evolution (LTE) and Worldwide Interoperability for Microwave Access (WiMax) are inevitable to coexist. Therefore, with the increasing amount of users and services, an effective load balancing approach in heterogeneous SGC networks is becoming extremely imperative.

Some previous literatures have addressed the heterogeneous SGC networks. The use of multiple RATs in a smart grid was first proposed in [1] to support the varying traffic requirements for overhead transmission line monitoring. At the network layer, architectures and frameworks to support such heterogeneous networks have been suggested in [2]-[4]. A survey of these architectures is provided in [2]. Recent proposals are based on the Media Independent Handover function defined by the IEEE 802.21 standard, which provides a framework to support seamless transition across networks based on different RATs [3]. Another relevant standard, IEEE P1900.4, defines building blocks for enabling coordinated network-device distributed decision making that contributes to the optimization of radio resource usage, including the spectrum access control, in heterogeneous wireless access networks [4]. Hierarchical resource managers have been proposed by the Common Radio Resource Management, Joint Radio Resource Management, and Multi-access Radio Resource Management schemes studied by the 3GPP group. And the emerging standards from the IETF and 3GPP communities to support flow mobility, when a device switches its data flows over multiple 
wireless access systems are proposed by [5]-[6]. However, none of the aforementioned literature puts forward an effective load balancing method for multiple RATs heterogeneous networks in smart grid.

In this paper, a context-aware load balancing scheme is proposed for heterogeneous SGC networks. Both the fairness and the overall throughput are investigated for demonstrate the enhancement of the proposed approach. The optimal radio resource allocation is determined for exploring the resources from either cellular base station (BS) or access point (AP).

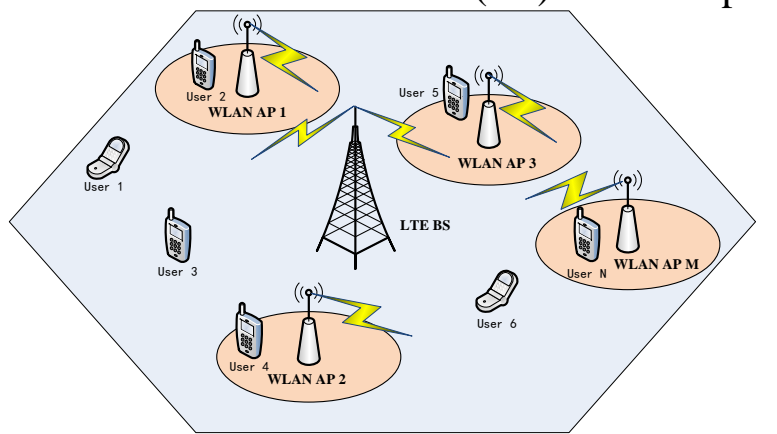

Figure 1. Heterogeneous Network Model in SGC

\section{System Model}

\section{A. Network Model}

The heterogeneous SGC network model under our consideration is depicted in Figure 1, which consists of one LTE cellular BS and several WLAN APs. And all the users are located in the region covered by LTE BS and WLAN Aps, which can only connect to one RAT simultaneously. The users of different kinds of service in the overlapping area can access to both RATs, which would make a big difference on the load condition of the network if users make different selection. To acquire the services effectively, each user should obtain the corresponding physical resource blocks (PRB) from BS or APs. Moreover, in order to ensure the quality of service (QoS) of each user, a minimum data rate is set for each service. In addition, we assume that different PRBs lie in different frequency bands, thus the interference between any of two PRBs could be ignored.

B. Context Information

In order to manage PRB in heterogeneous networks across smart grid efficiently, we need the relevant context information from both LTE BS and WLAN APs. We consider the following context information in our model.

1) LTE BS Load Condition: We assume that the average received signal to interference plus noise ratio (SINR) for user from cell $c$ at time $t \operatorname{SINR}_{u}$ is determined by the average channel gain, which is written as

$$
\operatorname{SINR}_{\mathrm{u}}=\frac{p_{c} \cdot L_{u}\left(\overrightarrow{p_{u}}\right)}{N+\sum_{c \neq X(u)} \rho_{c} \cdot p_{c} \cdot L_{u}\left(\overrightarrow{p_{u}}\right)}
$$

where $L_{u}\left(\overrightarrow{p_{u}}\right)$ is the average pathloss with shadow fading from the currently serving cell and other interference cell, $p_{c}$ denotes the transmit power for cell $c, \rho_{c}$ represents the load of the cell $C$ and $N$ is the power of additive white gaussian noise, respectively. Given the $\operatorname{SINR}_{u}$, we find the data rate per PRB by using the Shannon formular. Assuming that the adaptive coding and modulation is used to chieve the Shannon rate limit, the quantity of resources allocated to user $u$ by cell $j$ can be written by

$$
N_{\mathrm{u}}=\frac{D_{u}}{R(\operatorname{SINR}) \cdot B}
$$

where $R(\operatorname{SINR})=\log _{2}\left(1+\operatorname{SINR}_{u}\right), D_{u}$ represents the bit rate of user $u$ and $B$ is the bandwidth of per PRB. Therefore, we can acquire the total load of cell $c$ by

$$
\rho_{c}=\sum_{X(u)=c} \rho_{u}=\sum_{X(u)=c} \frac{D_{u}}{N_{\text {total }} \cdot R\left(\operatorname{SINR} R_{u}\right) \cdot B}
$$


2) WLAN AP Load Condition: We assume that the maximum capacities of differnet WLAN APs are identtical. In this paper, the load of WLAN AP $\rho_{w}$ is defined as the ratio of the number of current users accessing to WLAN AP $\left(U_{m}\right)$ to the maximum number of users that can access to the WLAN AP $\left(U_{\max }\right)$. The load is presented as

$$
\rho_{\mathrm{W}}=\frac{U_{m}}{U_{\max }}
$$

3) WLAN AP Connectivity Index: The connectivity index is to measure whether the users in the coverage of the WLAN hotspots is appropriate to having access to the WLAN AP. Large connectivity index indicates that users appropriate to access to WLAN APs. The connectivity index includes 2 parts: the mobile index and the ratio of received signal power of WLAN APs. The latter is that users received signal power compared to the maximum input signal power lever of user from WLAN hotspots. We assume that the maximum input signal power levels of user are the same to all users. The formula of the connectivity index can be expressed as

$$
k_{m, t}^{u}=\gamma_{1} \cdot \gamma_{m, t}^{u}+\gamma_{2} \cdot \frac{p_{m, t}^{u}}{p_{\max }}\left(\gamma_{1}+\gamma_{2}=1, p_{m, t}^{u}>p_{t}\right)
$$

where $p_{m, t}^{u}$ represents the received power strength in time slot $t$ from the WLAN AP $m, p_{t}$ is the received power threshold, $p_{\max }$ denotes the maximum input signal level that received by users, which is a constant, and $\gamma_{1}, \gamma_{2}$ represent the weight factors of mobile index and the received WLAN signal power ratio, respectively. The higher connectivity index of user $u$ indicates the user is proper to access to the WLAN AP; otherwise, the user is proper to access to the LTE BS.

\section{Problem Formulation}

\section{A. Load Balancing Index}

Load balancing aims to achieve a fair distribution of users among cells. It is desirable to have a method or an index that can be used to describe the degree of fairness. Typically, Jain's fairness index is used as a metric to assess the level of load balancing in a network. Since Jain's fairness index is used as a load balancing standard in the homogeneous network, we use it to measure the load fairness among different WLAN hotspots. The Jain's fairness index is indicated by

$$
\beta=\frac{\left(\sum \rho_{j}\right)^{2}}{n \sum\left(\rho_{j}\right)^{2}} \text {. }
$$

In order to make the load well-distributed in the two networks, we use $\varepsilon$ which represents the ratio of the two network load to measure the difference. We aim to make the $\varepsilon$ close to 1 , the $\varepsilon$ can be written as

$$
\varepsilon=\frac{\rho_{\mathrm{WLAN}}}{\rho_{\mathrm{c}}}=\frac{\sum_{n} U_{m}}{n U_{\text {max }}} .
$$

\section{B. Adaptive Balancing Threshold}

We use $\delta$ as the load balancing threshold. If $\rho_{\text {LTE }}-\rho_{\text {WLAN }} \geq \delta$, LTE BS load compared to WLAN AP is too heavy, thus a load balancing process is required to convert the LTE BS load to WLAN AP. If $\rho_{\text {WLAN }}-\rho_{\text {LTE }} \geq \delta$, which means that WLAN AP load compared to LTE BS is too heavy, we need to balance the WLAN AP.

For a dynamical network, a fixed balance threshold obviously cannot ensure the optimization performance of the network all the time. So we need the $\delta$ change itself versus the load condition. In this paper, we propose a heuristic algorithm. The load balancing threshold $\delta$ starts at a given value. When a load balancing process has been completed and the condition of $\left|\rho_{\text {LTE }}-\rho_{\text {WLAN }}\right|<\delta$ has not been met, the load balance threshold $\delta$ becomes $a \delta$, thus another load balancing process is required. Here $\alpha$ have two values. When $\delta_{\max }$ is met, $\alpha$ is the small value. When $\delta_{\min }$ is met, $\alpha$ is the larger one. 
These settings are made because they could make a limit to $\delta, \delta \in\left(\delta_{\min }, \delta_{\max }\right)$, so as to ensure the rationality of load balancing.

C. Mathematical Model

In order to improve the load condition of heterogeneous networks in smart grid, we need to enhance the load ratio and fairness, which will help improve the throughput of the heterogeneous networks. Therefore, we can formulate our problem in the following mathematical model.

$$
\begin{cases}\max & \beta=\frac{\left(\sum \rho_{j}\right)^{2}}{n \sum\left(\rho_{j}\right)^{2}} ; \quad \varepsilon=\frac{\rho_{\mathrm{WLAN}}}{\rho_{\mathrm{LTE}}} \\ \text { s.t. } & \mathrm{C}_{1}:\left|\rho_{\mathrm{LTE}}-\rho_{\mathrm{WLAN}}\right| \leq \delta \\ & \mathrm{C}_{2}: \sum_{X(u)=c} N_{u}<N_{\text {total }} \\ & \mathrm{C}_{3}: U_{m}<U_{\max } \quad \forall m \in[1,2, \cdots, M]\end{cases}
$$

In this mathematical model, constraint C1 means the difference between the LTE BS load and WLAN AP load is smaller than $\delta$, so the load can be distributed uniformly in the heterogeneous networks. Further, constraint C2 denotes the number of PRB allocated to users is smaller than $N_{\text {total }}$, which is the total number of PRB. Moreover, $U_{m}$ is the number of users accessing WLAN AP and $U_{\max }$ is the maximum number of users that an AP can serve. Constraint C3 ensures the number of the users served by each AP is smaller than $U_{\max }$.

\section{Context-aware Load Balancing Algorithm}

According to the discussion above, we can propose a context-aware algorithm to solve the load balancing problem in heterogeneous networks across smart grid. Through our proposed algorithm, we can effectively achieve the load balancing in heterogeneous networks. The outline of the proposed algorithm is depicted in Figure 2.

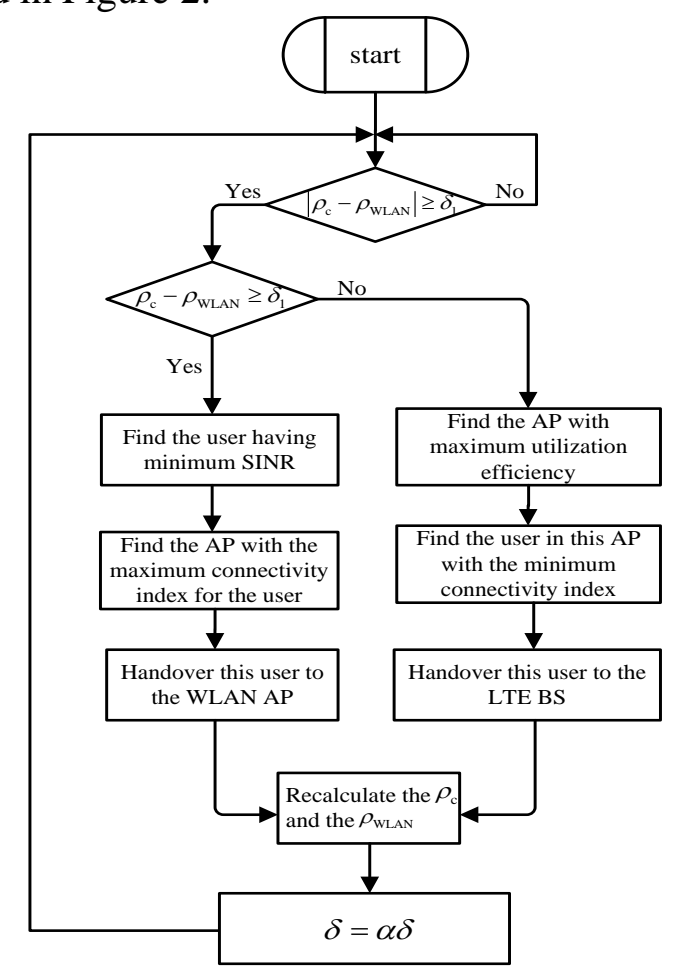

Figure 2. Context-aware Load Balancing Algorithm

\section{Simulation Results}

In order to validate our proposed algorithm, we need to simulate a practical scenario. In our scenario, there is one LTE BS and four WLAN APs located in the covered area. Meanwhile, we 
assume that the signal loss between the LTE BS and users including path fading and frequency selective fading can be calculated by $128+37 \lg (d)+21 \lg (1.3)(\mathrm{dB})$, and the path loss model between the users and the WLAN AP is $140.7+37.6 \lg (d)+21 \lg (1.3)(\mathrm{dB})$. Moreover, the simulation parameters of the system are shown in TABLE I.

TABLE I. SiMULATION PARAMETERS

\begin{tabular}{|c|c|c|c|}
\hline parameter & value & parameter & value \\
\hline The coverage radius of LTE BS & $500 \mathrm{~m}$ & Power Spectrum Density of Noise & $180 \mathrm{dBm}$ \\
\hline Transmit power of LTE BS & $40 \mathrm{~W}$ & WLAN bandwidth & $22 \mathrm{MHZ}$ \\
\hline Number of Resource blocks & 100 & Number of WLAN APs in a cell & 4 \\
\hline LTE Resource block Bandwidth & $180 \mathrm{KHZ}$ & The coverage radius of WLAN & $40 \mathrm{~m}$ \\
\hline Number of cells in a system & 7 & Transmit power of WLAN AP & $0.1 \mathrm{~W}$ \\
\hline Shadow standard deviation & $8 \mathrm{~dB}$ & $\begin{array}{c}\text { Maximum user number of each } \\
\text { WLAN AP }\end{array}$ & 10 \\
\hline
\end{tabular}

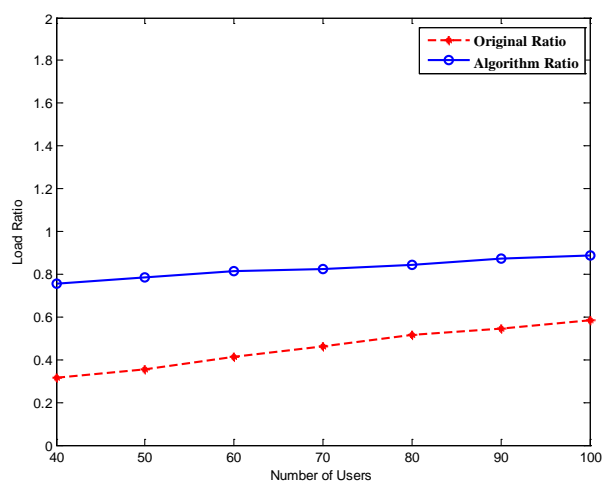

Figure 3. Load Ratio in heterogeneous networks.

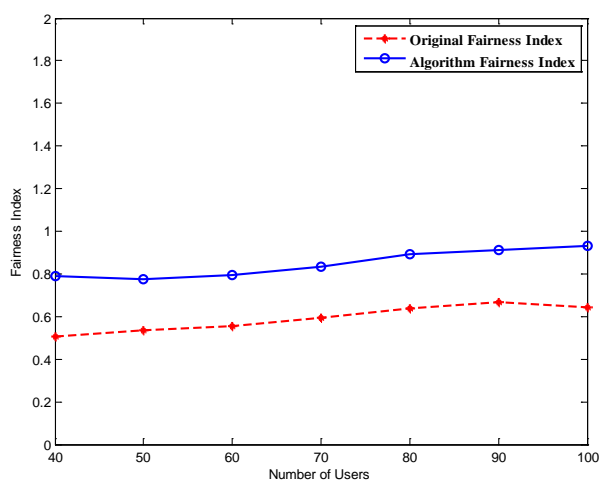

Figure 4. Fairness Index in heterogeneous networks.

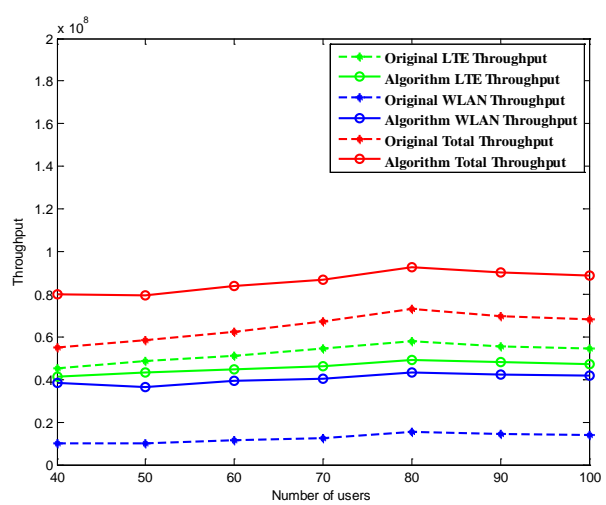

Figure 5. Throughput in heterogeneous networks. 
Figure 3 shows the ratio of the average WLAN AP load to LTE BS load in heterogeneous SGC networks. Before the load balancing algorithm is applied, the fairness $\beta$ in heterogeneous networks is about 0.4 . Through our proposed load balancing algorithm, $\beta$ can enhance to 0.9 approximately, which indicates that the load between LTE BS and WLAN APs is balanced since some users have been transferred to the WLAN APs.

Figure 4 indicates the fairness index in networks. With the increasing of users, the original index is around 0.6. By our proposed algorithm, the fairness index in the networks can be improved up to 0.9. Therefore, this implies that the proposed algorithm can balance the load condition among LTE BS and different WLAN APs.

The throughput of the heterogeneous networks is shown in Figure 5. As the number of users in the LTE BS increases, the network total throughput gradually increases. When the number of users is around 80, the total throughput is maximized, where the proposed algorithm has improved the performance by $30 \%$. After the load balancing algorithm, the WLAN throughput increases while the LTE throughput decreases, which is due to the fact that some users have been transferred to the WLAN APs from LTE BS.

\section{Conclusions}

In this paper, a context-aware load balancing scheme was proposed in heterogeneous SGC networks. By the proposed algorithm, we can improve the load condition of heterogeneous SGC networks effectively with sufficient context information. Through the network optimization realized by our proposed algorithm, we can acquire a higher total network throughput, which guarantees a better environment for smart grid communication.

\section{References}

[1]K. Hung et. al, "On Wireless Sensor Communication for Overhead Transmission Line Monitoring in Power Delivery Systems,” Proceedings of IEEE SmartGridComm, Oct. 2010.

[2]L. Gavrilovska, V. Atanasovski, "Resource Management in Wireless Heterogeneous Networks (WHNs),” Telsiks October 2009.

[3]IEEE Std 802.21-2008, IEEE Standard for Local and Metropolitan Area Networks, Part 21: Media Independent Handover Services, IEEE, Jan. 2009.

[4]S. Buljore et. al., "Architecture and Enablers for Optimized Radio Resource usage in Heterogeneous Wireless Access Networks: The IEEE 1900.4 Working Group,” IEEE Communications Magazine, Jan. 2009.

[5]A. Oliva, C. Bernardos, M. Calderon, T. Melia, and J. Zuniga, “IP Flow Mobility: Smart Traffic Offload for Future Wireless Networks,” IEEE Communications Magazine, vol. 49, no. 10, Oct 2011.

[6]3rd Generation Partnership Project, “Architecture enhancements for non-3GPP accesses,” 3GPP TS 23.402, 2011. 